\title{
Estimating skin blood saturation by selecting a subset of hyperspectral imaging data
}

\author{
Maria Ewerlöf, E. Göran Salerud, Tomas Strömberg and Marcus Larsson
}

Linköping University Post Print

Tweet

N.B.: When citing this work, cite the original article.

Original Publication:

Maria Ewerlöf, E. Göran Salerud, Tomas Strömberg and Marcus Larsson, Estimating skin blood saturation by selecting a subset of hyperspectral imaging data, 2015, Imaging, Manipulation, and Analysis of Biomolecules, Cells, and Tissues XIII, 93280J (March 2). http://dx.doi.org/10.1117/12.2075292

Copyright: (C) 2015 SPIE

http://proceedings.spiedigitallibrary.org/

Postprint available at: Linköping University Electronic Press

http://urn.kb.se/resolve?urn=urn:nbn:se:liu:diva-116478 


\title{
Estimating skin blood saturation by selecting a subset of hyperspectral imaging data
}

\author{
Maria Ewerlöf, E. Göran Salerud, Tomas Strömberg, Marcus Larsson \\ Department of Biomedical Engineering, Linköping University, S-581 83 Linköping, Sweden
}

\begin{abstract}
Skin blood haemoglobin saturation $\left(s_{\mathrm{b}}\right)$ can be estimated with hyperspectral imaging using the wavelength $(\lambda)$ range of 450-700 $\mathrm{nm}$ where haemoglobin absorption displays distinct spectral characteristics. Depending on the image size and photon transport algorithm, computations may be demanding. Therefore, this work aims to evaluate subsets with a reduced number of wavelengths for $s_{\mathrm{b}}$ estimation.
\end{abstract}

White Monte Carlo simulations are performed using a two-layered tissue model with discrete values for epidermal thickness $\left(T_{\text {epi }}\right)$ and the reduced scattering coefficient $\left(\mu_{\mathrm{s}}^{\prime}\right)$, mimicking an imaging setup. A detected intensity look-up table is calculated for a range of model parameter values relevant to human skin, adding absorption effects in the post-processing. Skin model parameters, including absorbers, are; $\mu_{\mathrm{s}}^{\prime}(\lambda), T_{\text {epi }}$, haemoglobin saturation $\left(s_{\mathrm{b}}\right)$, tissue fraction blood $\left(c_{\mathrm{b}}\right)$ and tissue fraction melanin $\left(c_{\text {mel }}\right)$. The skin model paired with the look-up table allow spectra to be calculated swiftly.

Three inverse models with varying number of free parameters are evaluated: $\mathrm{A}\left(s_{\mathrm{b}}, c_{\mathrm{b}}\right), \mathrm{B}\left(s_{\mathrm{b}}, c_{\mathrm{b}}, c_{\mathrm{mel}}\right)$ and $\mathrm{C}$ (all parameters free). Fourteen wavelength candidates are selected by analysing the maximal spectral sensitivity to $s_{\mathrm{b}}$ and minimizing the sensitivity to $c_{\mathrm{b}}$. All possible combinations of these candidates with three, four and 14 wavelengths, as well as the full spectral range, are evaluated for estimating $s_{\mathrm{b}}$ for 1000 randomly generated evaluation spectra.

The results show that the simplified models A and B estimated $s_{\mathrm{b}}$ accurately using four wavelengths (mean error $2.2 \%$ for model B). If the number of wavelengths increased, the model complexity needed to be increased to avoid poor estimations.

Keywords: Hyper spectral imaging; skin blood saturation; diffuse reflectance spectroscopy; Monte Carlo; skin optical properties; computer modelling and simulation; microcirculation

\section{INTRODUCTION}

All living tissue consumes oxygen and this is a fundamental and powerful indicator of the health of any organ. The carrier of oxygen to the tissue, the haemoglobin, readily absorbs it in the lungs, and releases it at low partial pressures to nurture the tissue ${ }^{1}$. Its spectral signatures throughout the visible spectrum easily identify the two dominant forms of haemoglobin, oxygenated and deoxygenated haemoglobin ${ }^{2}$. The extraction of oxygen to the tissue, or the tissue oxygenation, can be monitored by analysing the spectral signatures for determining the relation between concentrations of oxygen carrying haemoglobin to the total amount of haemoglobin ${ }^{3}$.

The most common technique to determine the concentrations of the tissue chromophores and the oxygenation, diffuse reflectance spectroscopy (DRS), utilize optical fiber probes with a well-defined distance between sending and receiving fibers ${ }^{4}$. DRS measures the light intensity from skin tissues as a function of wavelength, and the spectral acquisition and decomposition return quantitative information about its biochemical properties ${ }^{5}$. Therefore, a theoretical model outlining the diffuse reflectance spectra due to light-tissue interactions is needed ${ }^{6}$. Monte Carlo simulations ${ }^{7-9}$, Kubelka-Munk ${ }^{10,11}$, and the modified Beer-Lambert law ${ }^{12}$ are the models used most frequently. Minimizing the difference between the inversely modelled diffuse reflectance spectrum and the measured reflectance spectrum, permit the unknown tissue parameters such as blood saturation to be estimated, based on a priori physical knowledge of skin absorption and scattering properties and thickness. 
Fiberoptic or pointwise based estimation of the tissue optical properties limits the spatial diagnostic abilities found by experienced clinicians. The spatial distribution of skin chromophores and oxygenation can be achieved by diffuse hyperspectral imaging (HSI). This can be obtained by combining advantages of the spectrophotometer and digital cameras. It can potentially provide information not detectable by the human eye and can lead to objective skin chromophore quantification. HSI, as an emerging imaging modality for medical applications, offers great potential for non-invasive disease diagnosis and surgical guidance. ${ }^{13}$ Advances in hyperspectral cameras, image analysis methods, and computational power make it possible for many clinically relevant applications in the medical field.

To accurately analyse the large HSI datasets for blood saturation quantification is computationally demanding. Depending on the light transport model used and the image size, it may be difficult to do in real time. To lower computational demands, reduced HSI datasets may be used that can be achieved using only a selected number of wavelengths (multispectral imaging, MSI) or by using multiple cameras with properly selected filters. The latter solution also allows for simultaneous recording of the spectrum, without the time delay during the wavelength selection and shifting in e.g. a liquid crystal tunable filter, and may also lower the hardware cost.

Therefore, this work aims to find sets with a reduced number of wavelengths where blood saturation in skin can be evaluated at a high precision and accuracy.

\section{METHODS}

\subsection{Skin tissue model}

A two-layered skin model is used and parameterized discretely. The upper and outermost layer, containing melanin but no blood, resembles the epidermis with a thickness $T_{\text {epi }}$ ranging from 50 to $200 \mu \mathrm{m} .{ }^{1,14,15}$ The second layer, assumed to be semi-infinite in relation to the optical properties, has a fixed thickness of $5 \mathrm{~cm}$, and contains blood. Both layers are assigned the same scattering properties, using a Henyey-Greenstein scattering phase function, with anisotropy factor $g=0.8$ for all wavelengths $\lambda$. The wavelength-dependent scattering coefficient, $\mu_{\mathrm{s}}^{\prime}(\lambda)$, is described by the parameters $\alpha$ and $\beta$ according to Eq. $1^{14}$.

$$
\mu_{\mathrm{s}}^{\prime}(\lambda)=\alpha \cdot\left(\frac{\lambda}{500}\right)^{-\beta}
$$

For the two-layered skin model $\alpha$ in the range from 3 to $6 \mathrm{~mm}^{-1}$ and $\beta$ in the range from 0.5 to 2.5 were considered relevant. ${ }^{14}$ Calculating $\mu_{\mathrm{s}}^{\prime}(\lambda)$ for combinations of $\alpha$ and $\beta$ within the defined range, resulted in $\mu_{\mathrm{s}}^{\prime}$ values between 1 and $10 \mathrm{~mm}^{-1}$, using a wavelength range of 450 to $700 \mathrm{~nm}$.

The absorption coefficient of the epidermal layer, $\mu_{\mathrm{a} \text {,mel }}(\lambda)$, is modelled in Eq. 2,

$$
\mu_{\mathrm{a}, \mathrm{mel}}(\lambda)=c_{\mathrm{mel}} \cdot 51.9 \cdot\left(\frac{\lambda}{500}\right)^{-3}
$$

with $c_{\text {mel }}$ as the tissue fraction of melanin. To cover most relevant values of $c_{\text {mel }}$, a range is set from 0 to $4.2 \%$, representing values from light Caucasian skin to darker skin. ${ }^{14}$ For the wavelength range 450 to $700 \mathrm{~nm}$ this results in $\mu_{\mathrm{a} \text {,mel }}$ values less than $3 \mathrm{~mm}^{-1}$.

The absorption coefficient due to blood, $\mu_{\mathrm{a}, \mathrm{b}}(\lambda)$, in the second layer is given by Eq. 3 ,

$$
\mu_{\mathrm{a}, \mathrm{b}}(\lambda)=\left(1-s_{\mathrm{b}}\right) \cdot c_{\mathrm{b}} \cdot 0.15 \cdot \mu_{\mathrm{a}, \mathrm{Hb}}(\lambda)+s_{\mathrm{b}} \cdot c_{\mathrm{b}} \cdot 0.15 \cdot \mu_{\mathrm{a}, \mathrm{HbO} 2}(\lambda)
$$

where $s_{\mathrm{b}}$ is the saturation level, $c_{\mathrm{b}}$ represent blood tissue fraction, $\mu_{\mathrm{a}, \mathrm{Hb}}$ the absorption coefficient of deoxygenated haemoglobin, and $\mu_{\mathrm{a}, \mathrm{HbO} 2}$ the absorption coefficient of oxygenated haemoglobin ${ }^{2}$. A $15 \%$ haemoglobin fraction of the blood is assumed and the saturation varies between $0-100 \%$ with a blood tissue fraction between $0-1.4 \%$. For the wavelength range 450 to $700 \mathrm{~nm}$ this results in $\mu_{\mathrm{a}, \mathrm{b}}$ values less than $0.5 \mathrm{~mm}^{-1} .{ }^{14}$ 


\subsection{Monte Carlo simulations}

To model photon propagation in the two-layered tissue model, a number of base simulations are run for 37 equidistant levels of $\mu_{\mathrm{s}}^{\prime}$ between 1 and $10 \mathrm{~mm}^{-1}$. For each of the $37 \mu_{\mathrm{s}}^{\prime}$ levels three epidermal thicknesses $(50,100$ and $200 \mu \mathrm{m})$ where evaluated, i.e. in total 111 simulations. Simulations are performed using White Monte Carlo and absorption is therefore added in the post processing.

In each simulation, photons are injected perpendicularly into the tissue from a pencil beam until the number of detected backscattered photons $\left(N_{\text {det }}\right)$ at the top layer reaches 200000 counts. The path lengths in each of the two layers, $p l_{1, \mathrm{n}}$ and $p l_{2, \mathrm{n}}$, for each detected photon $(n)$ as well as the total number of emitted photons $\left(N_{\mathrm{em}}\right)$ are stored to include the effect of absorption in the post processing. All simulations were performed with an in-house Monte Carlo simulation software [9] that have been extensively used and validated.

\subsection{Post processing}

Absorption was added to each base simulation using Beer-Lambert's law and the path lengths in the epidermal layer, $p l_{1}$, and the second layer, $p l_{2}$, for each detected photon $(n)$ in accordance with Eq. 4 where $N_{\text {det }}$ is the number of detected photons. To compare simulations $N_{\text {det }}$ was normalized by the total number of emitted photons $N_{\text {em }}$ for every base simulation.

$$
I_{\text {norm }}=\frac{1}{N_{\mathrm{em}}} \sum_{n=1}^{N_{\mathrm{det}}} e^{-\mu_{\mathrm{a}, \mathrm{mel}} \cdot p l_{1, \mathrm{n}} \cdot e^{-\mu_{\mathrm{a}, \mathrm{b}} \cdot p l_{2, \mathrm{n}}}}
$$

For a faster inverse simulation process that is less computationally demanding, a look-up table was created where the total backscattered intensity, $I_{\mathrm{M}}$, and thereby the spectrum for any value of $\mu_{\mathrm{s}}^{\prime}, \mu_{\mathrm{a}, \mathrm{mel}}, \mu_{\mathrm{a}, \mathrm{b}}$ and $T_{\mathrm{epi}}$, could be extracted. The above calculations were performed for 21 levels of $\mu_{\mathrm{a}, \mathrm{mel}}$ equidistantly distributed between 0 and $3 \mathrm{~mm}^{-1}$ and for 101 levels of $\mu_{\mathrm{a}, \mathrm{b}}$ equidistantly distributed between 0 and $0.5 \mathrm{~mm}^{-1}$. The resulting intensities $I_{\text {norm }}$ were saved in the matrix $I_{\mathrm{LUT}}\left(\mu_{\mathrm{s}}^{\prime}, \mu_{\mathrm{a}, \mathrm{mel}}, \mu_{\mathrm{a}, \mathrm{b}}, T_{\mathrm{epi}}\right)$ for further use.

\subsection{Spectra calculations}

The skin tissue model is defined by six parameters, describing tissue scattering (parameter $\alpha$ and $\beta$ ), tissue fraction melanin $\left(c_{\text {mel }}\right)$, tissue fraction blood $\left(c_{\mathrm{b}}\right)$, blood saturation $\left(s_{\mathrm{b}}\right)$ and epidermal thickness $\left(T_{\text {epi }}\right)$. In a given wavelength interval, these parameters can be used to calculate the tissue optical properties $\mu_{\mathrm{s}}^{\prime}(\lambda), \mu_{\mathrm{a}, \mathrm{mel}}(\lambda)$ and $\mu_{\mathrm{a}, \mathrm{b}}(\lambda)$. These properties, together with $T_{\text {epi }}$, are then used to generate simulated diffuse reflectance spectra using linear interpolation within the lookup-table, $I_{\mathrm{LUT}}$, dataset. This enables a swift process to generate simulated spectra for any combination of parameters confined to their respective interval (Table 1).

Table 1. The six parameters in the skin tissue model and their ranges. For the inverse Monte Carlo models (A, B and C), the values of the bound parameters are given by their interval midpoint.

\begin{tabular}{|c|c|c|c|c|}
\hline Parameter & Value range & Model A & Model B & Model C \\
\hline$\alpha$ & {$[3,6]$} & 4.5 & 4.5 & free \\
\hline$\beta$ & {$[0.5,2.5]$} & 1.5 & 1.5 & free \\
\hline$c_{\mathrm{mel}}$ & {$[0,0.042]$} & 0.021 & free & free \\
\hline$c_{\mathrm{b}}$ & {$[0,0.014]$} & free & free & free \\
\hline$s_{\mathrm{b}}$ & {$[0,1]$} & free & free & free \\
\hline$T_{\mathrm{epi}}$ & {$[50,200]$} & 125 & 125 & free \\
\hline
\end{tabular}




\subsection{Inverse Monte Carlo}

Three inverse Monte Carlo models, all based on the skin model presented above but with different degrees of freedom, are evaluated: In model A, the two parameters $s_{\mathrm{b}}$ and $c_{\mathrm{b}}$ are free in the fitting process; in model B the additional parameter $c_{\text {mel }}$ is set free; while all six parameters are free in model C. For the reduced models A and B, all other parameters are set to their respective interval midpoint (Table 1).

The inverse Monte Carlo calculations are performed with a trust-region-reflective algorithm (MATLAB version 8.2.0, 2013b, computer software, The MathWorks Inc., Natick, Massachusetts). The algorithm finds the optimal fit between a simulated evaluation spectrum, $I_{\mathrm{E}}(\lambda)$, and a modelled spectrum, $I_{\mathrm{M}}(\lambda)$, spectra generated from the $I_{\mathrm{LUT}}$ table as described in Sec. 2.4. The optimal fit is found by solving Eq. 5,

$$
\min _{x}\left\|\frac{I_{\mathrm{M}}(\lambda, x)}{\left\langle I_{\mathrm{M}}(\lambda, x)\right\rangle_{\lambda}} \frac{\left\langle I_{\mathrm{E}}(\lambda)\right\rangle_{\lambda}}{I_{\mathrm{E}}(\lambda)}-1\right\|_{2}^{2}
$$

where the parameter list $x$ is $\left[c_{\mathrm{b}}, s_{\mathrm{b}}\right]$ for model $\mathrm{A},\left[c_{\mathrm{b}}, s_{\mathrm{b}}, c_{\text {mel }}\right]$ for model B and $\left[c_{\mathrm{b}}, s_{\mathrm{b}}, c_{\text {mel }}, \alpha, \beta, T_{\text {epi }}\right]$ for model C, respectively. The penalty function is designed to be sensitive only to the spectral shape and not the absolute magnitude, making the results valid for a camera application where absolute calibration is virtually impossible. As a consequence, the minimum number of wavelengths needed is one more than the number of free parameters.

Each model is evaluated for a range of different wavelength combinations, as described in Sec. 2.6. A set of 1000 simulated evaluation spectra is generated by using the method described in Sec. 2.4 with parameter values randomly chosen within their respective range. Each of these combinations, is analysed in order to determine how well the optimized $\hat{s}_{\mathrm{b}}$ parameter matches the blood saturation level, $s_{\mathrm{b}}$, of the generated evaluation spectrum. To avoid ill conditioned problems, minimum blood amount was set to $0.1 \%$ when generating evaluation spectra. The modelled spectra, that are recursively updated in the optimization process, are however allowed a minimum blood amount of $0 \%$.

\subsection{Selecting wavelengths}

To limit the number of wavelengths to evaluate, we perform a small study to find what wavelengths are most sensitive to changes in blood saturation. This was done by comparing results from simulations for fully saturated blood (100\%) and de-saturated blood $(0 \%)$, with all other parameters set to the midpoint value of their defined interval, and calculating the ratio between the spectra. From Fig. 1 we find three maximum peaks (515, 560 and $598 \mathrm{~nm}$ ), three troughs (466, 538 and $578 \mathrm{~nm}$ ) and five isobestic points $(506,523,549,570$ and $586 \mathrm{~nm})$. We additionally select three points with low sensitivity to blood amount (i.e. a ratio close to one). These points are selected based on the ratio between simulated spectra using a maximal blood tissue fraction $(1.4 \%)$ and a low blood tissue fraction $(0.1 \%)$, while all other parameters are set to the midpoint value of their respective intervals. This resulted in three wavelengths; 484, 630 and $700 \mathrm{~nm}$ (Fig. 2).

Three different sizes of selected wavelength combinations are evaluated; three, four and 14 (all selected). Within each of these wavelength subsets, all possible combinations of selected wavelengths are evaluated. In addition, a set of all 251 wavelengths (from 450 to $750 \mathrm{~nm}$, in steps of $1 \mathrm{~nm}$ ) is evaluated. However, the need to add one extra wavelength in comparison to the number of free parameters means that model A can be evaluated for all four alternatives, model B for all but three wavelengths and model $\mathrm{C}$ only for 14 and 251 wavelengths. For each model (A, B and C) every combination of the current number of wavelengths is evaluated.

\subsection{Finding optimal wavelength combinations}

To find wavelength combinations giving the best saturation estimate, the difference between the saturation value $\left(s_{\mathrm{b}}\right)$ used to generate each evaluation spectrum and the corresponding value from the fitting process $\left(\hat{s}_{\mathrm{b}}\right)$ is determined. The root mean square error $\left(\varepsilon_{\mathrm{rms}}\right)$ of the difference is calculated in Eq. 6,

$$
\varepsilon_{\mathrm{rms}}=\left(\left\langle\left(\hat{s}_{\mathrm{b}}-s_{\mathrm{b}}\right)^{2}\right\rangle_{N_{\mathrm{E}}}\right)^{1 / 2}
$$

where $N_{\mathrm{E}}=1000$ evaluation spectra, is evaluated for all wavelength combinations. 

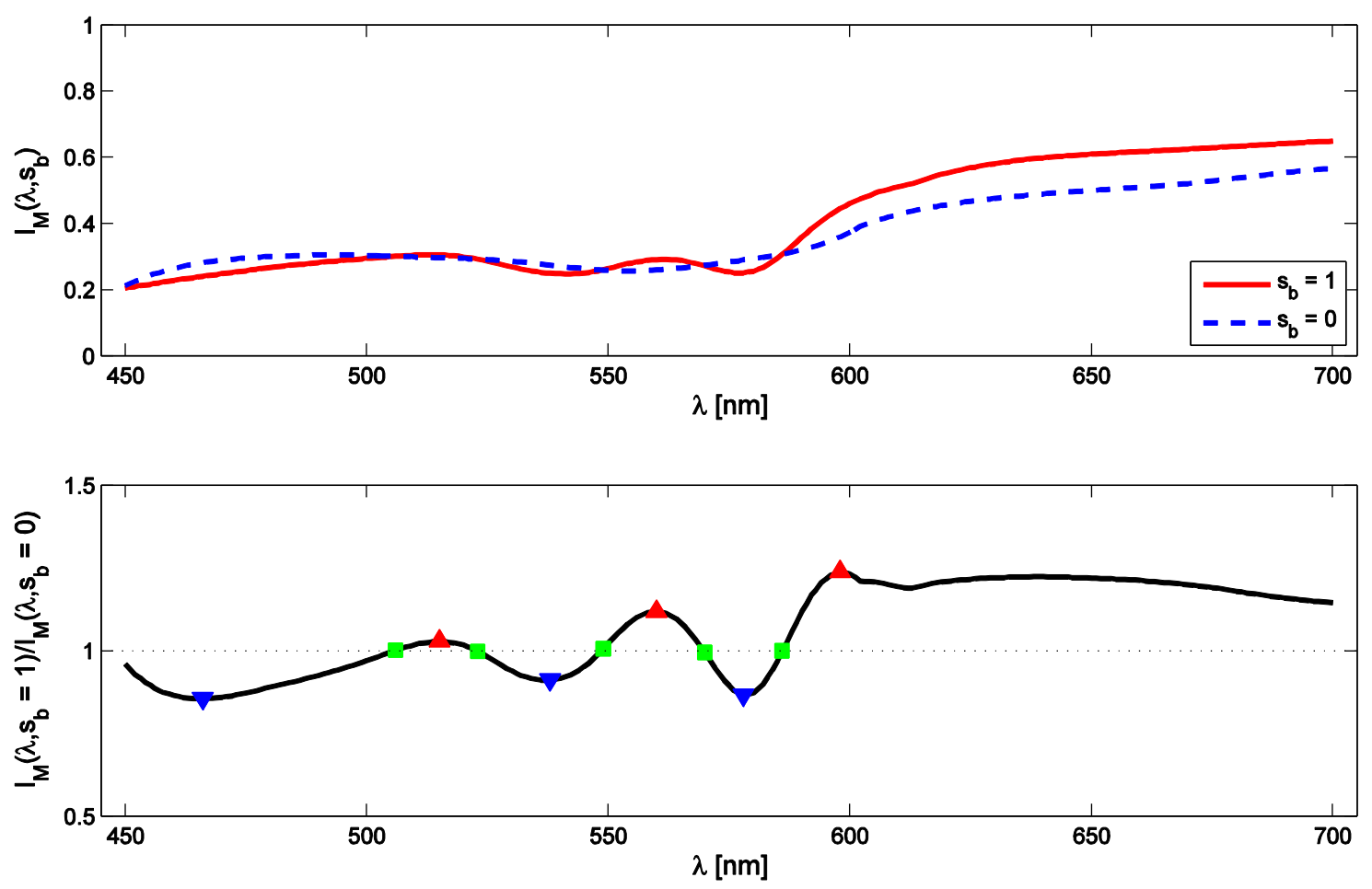

Fig. 1. Upper panel: Diffuse reflectance spectra for oxygenated blood $\left(s_{\mathrm{b}}=1\right)$ and fully deoxygenated blood $\left(s_{\mathrm{b}}=0\right)$. Lower panel: The ratio of the two spectra and the chosen wavelengths, (peaks $=$ red upward-pointing triangles, troughs $=$ blue downward-pointing triangles and isobestic points $=$ green squares.
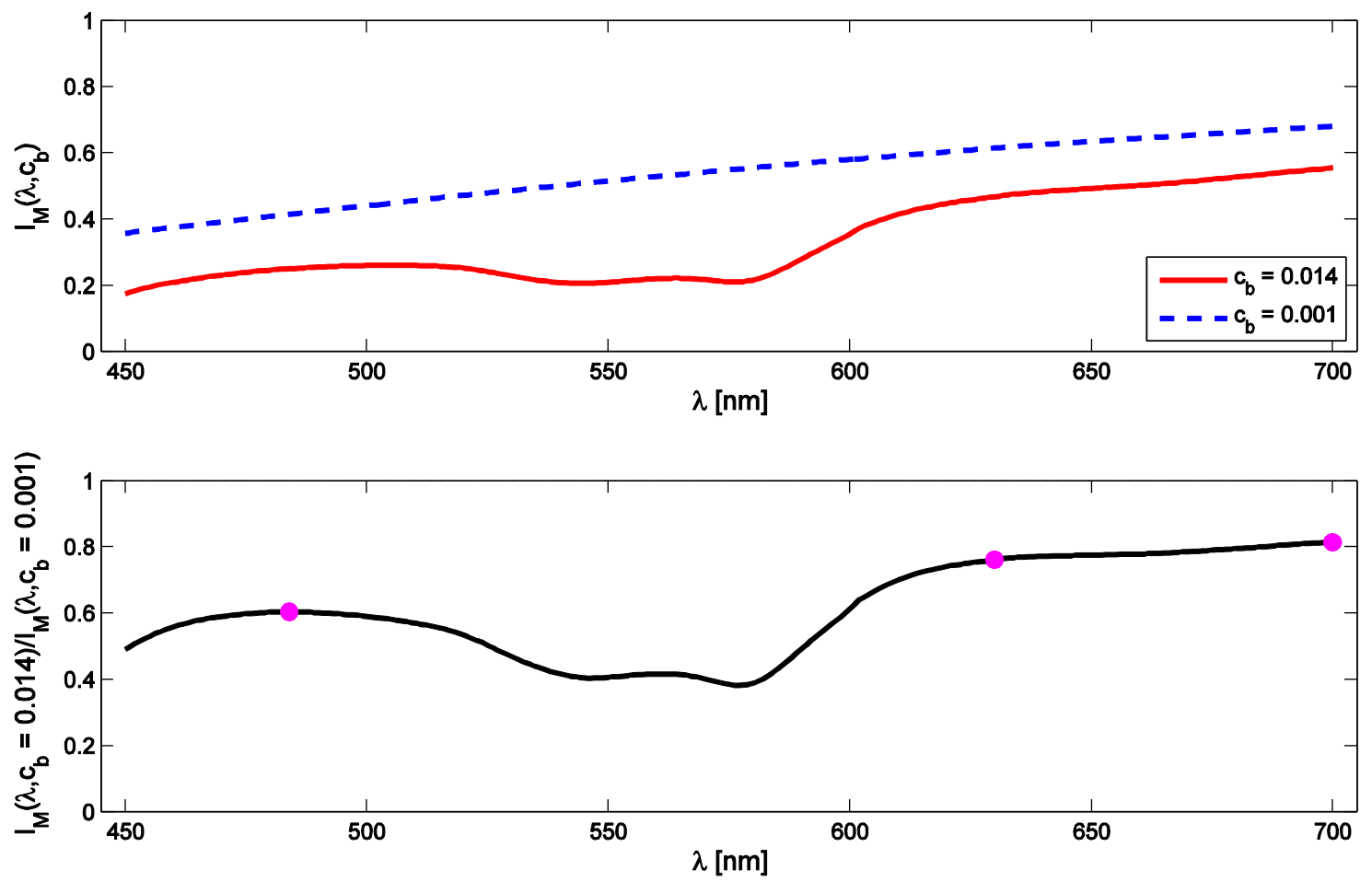

Fig. 2. Upper panel: Diffuse reflectance spectra for high $\left(c_{\mathrm{b}}=0.014\right)$ and low $\left(c_{\mathrm{b}}=0.001\right)$ blood tissue fraction. Lower panel: The ratio of the two spectra and the chosen wavelengths (magenta coloured dots). 


\section{RESULTS}

The lowest saturation estimation error, $\varepsilon_{\mathrm{rms}}$, found when evaluating all sets of possible wavelength combinations using model A, B and C, is presented in Table 2 . A representative spectrum $I_{\mathrm{E}}$ from the generated data set, with its corresponding modelled spectrum $I_{\mathrm{M}}$ for models A, B and C (251 wavelengths) having error values of $|\hat{s}-s|$ which are similar to their respective $\varepsilon_{\text {rms }}$ values is shown in Fig. 3. In Fig. 4, $I_{\mathrm{E}}$ is compared to the modelled spectrum for model B evaluated for four wavelengths (the wavelength combination with the lowest $\varepsilon_{\text {rms }}$ ).

In order to find the best combinations of our analysed wavelengths, the combinations with the ten lowest $\varepsilon_{r m s}$ values are chosen for model A (combinations of three and four wavelengths) and model B (four wavelengths), and presented in Figs. $5-7$.

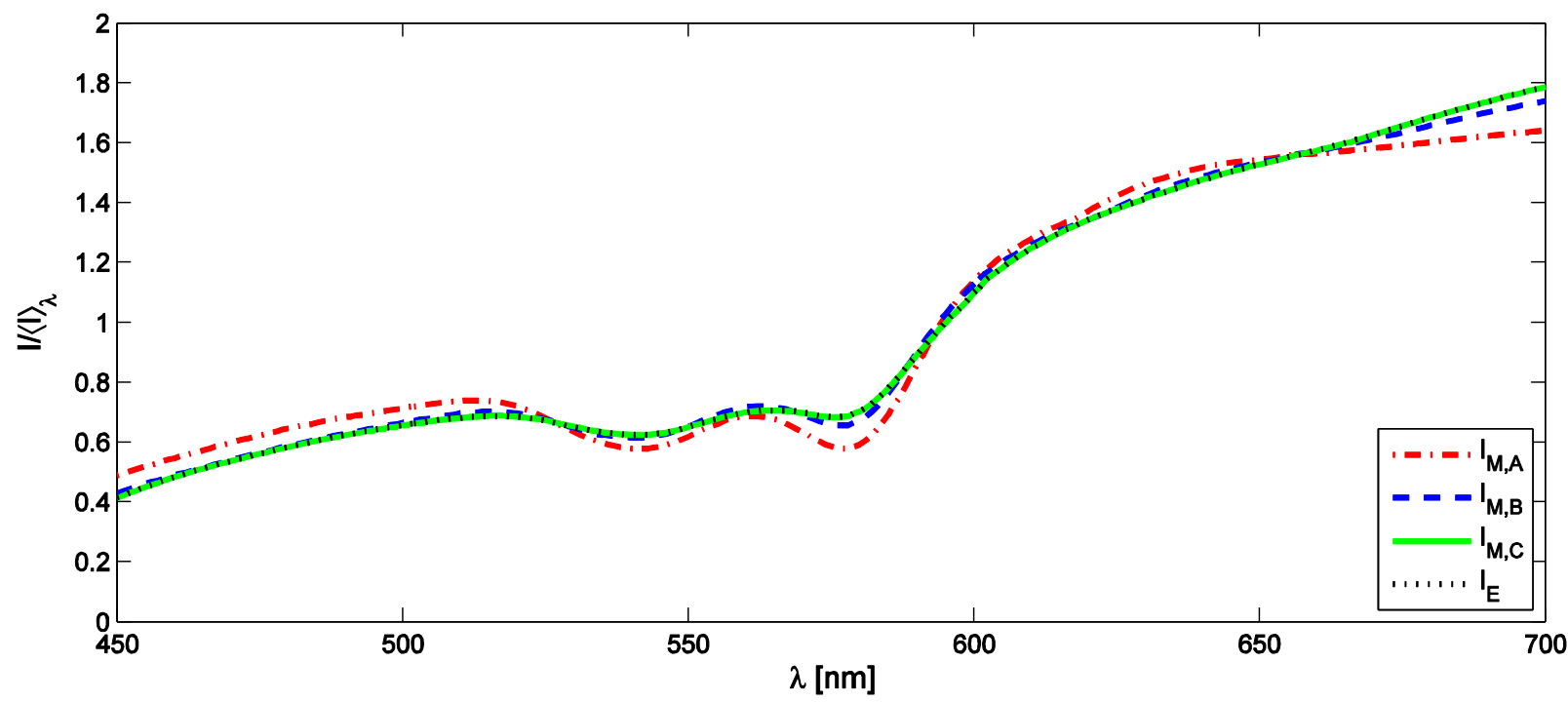

Fig. 3. A representative evaluation spectrum $\left(I_{\mathrm{E}}\right.$, black dotted line) and the corresponding spectra for model A ( $I_{\mathrm{M}, \mathrm{A}}$, red dash-dotted line), model B ( $I_{\mathrm{M}, \mathrm{B}}$, blue dashed line) and model C, $\left(I_{\mathrm{M}, \mathrm{C}}\right.$, green solid line), respectively. The modelling is done for 251 wavelengths. $\hat{s}-s=0.31,0.15$ and 0.021 for model A, B and C, respectively.

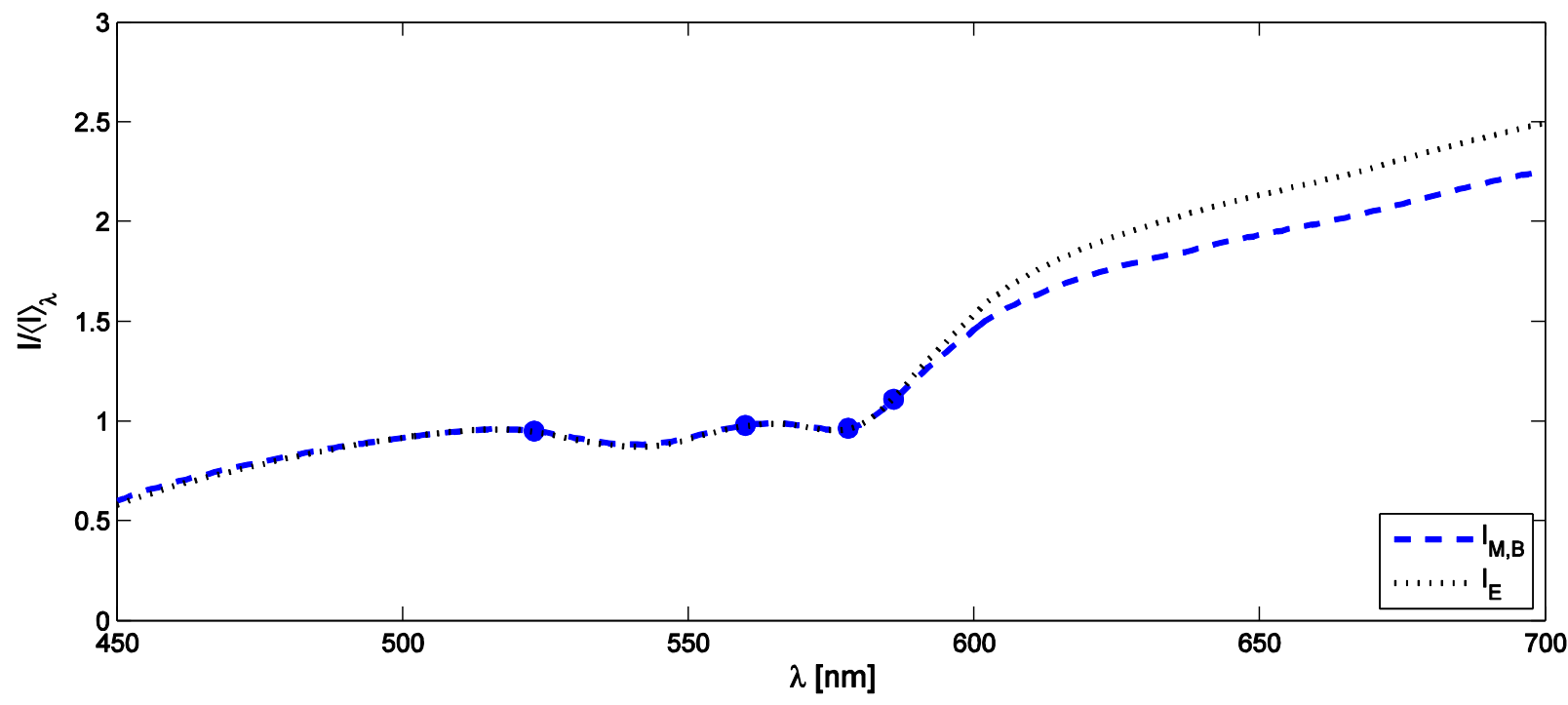

Fig. 4. A representative evaluation spectrum $\left(I_{\mathrm{E}}\right.$, black dotted line) and the corresponding modelled spectrum for model B ( $I_{\mathrm{M}, \mathrm{B}}$, blue dashed line). The modelling is done and normalized for four wavelengths (blue dots). $\hat{s}-s=0.018$. 
Table 2. The $\varepsilon_{\text {rms }}$ and its standard deviation (SD) over the 1000 evaluation spectra. For each model, the result for the wavelength combination with the lowest $\varepsilon_{\mathrm{rms}}$ is presented.

\begin{tabular}{|c|c|c|c|c|c|c|}
\hline \multirow{2}{*}{$\begin{array}{l}\text { Number of } \\
\text { wavelengths }\end{array}$} & \multicolumn{2}{|c|}{ Model A } & \multicolumn{2}{|c|}{ Model B } & \multicolumn{2}{|c|}{ Model C } \\
\hline & $\varepsilon_{\mathrm{rms}}$ & $\mathrm{SD}$ & $\varepsilon_{\mathrm{rms}}$ & $\mathrm{SD}$ & $\varepsilon_{\mathrm{rms}}$ & $\mathrm{SD}$ \\
\hline 3 & 0,069 & 0,0028 & & & & \\
\hline 4 & 0,040 & 0,0011 & 0,022 & 0,0012 & & \\
\hline 14 & 0,32 & 0,0047 & 0,086 & 0,0034 & 0,016 & 0,0005 \\
\hline 251 & 0,38 & 0,0051 & 0,10 & 0,0045 & 0,021 & 0,0013 \\
\hline
\end{tabular}
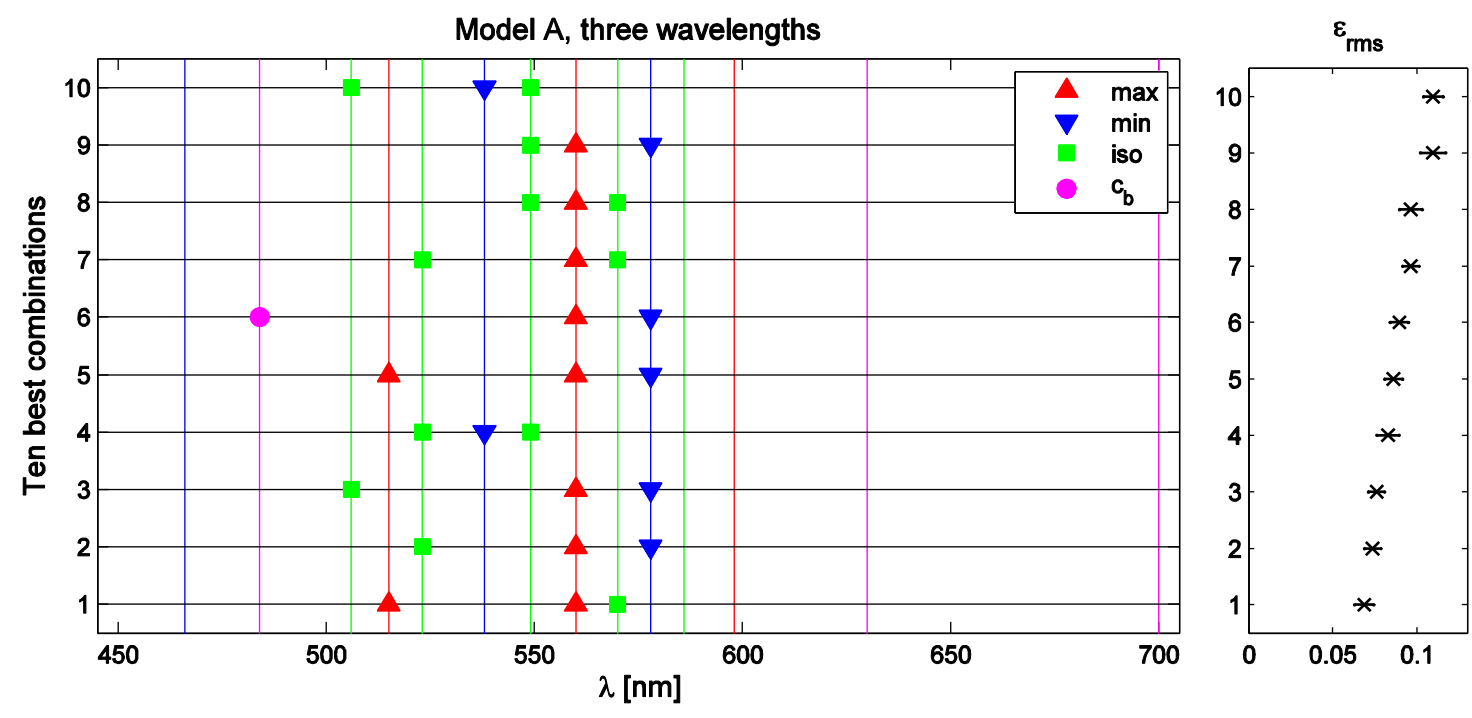

Fig. 5. The ten three-wavelength combinations with the lowest $\varepsilon_{\text {rms }}$ for model A. The vertical lines represent the 14 chosen wavelengths, the symbols on each horizontal line showing the three tested wavelengths. For the wavelength symbols, red upward-pointing triangle, blue downward-pointing triangle and the green square, see Fig. 1, for the magenta coloured dot, see Fig. 2. The $\varepsilon_{\text {rms }}$ for each threewavelength combination $\pm 2 \mathrm{SD}$ is shown in the right panel.
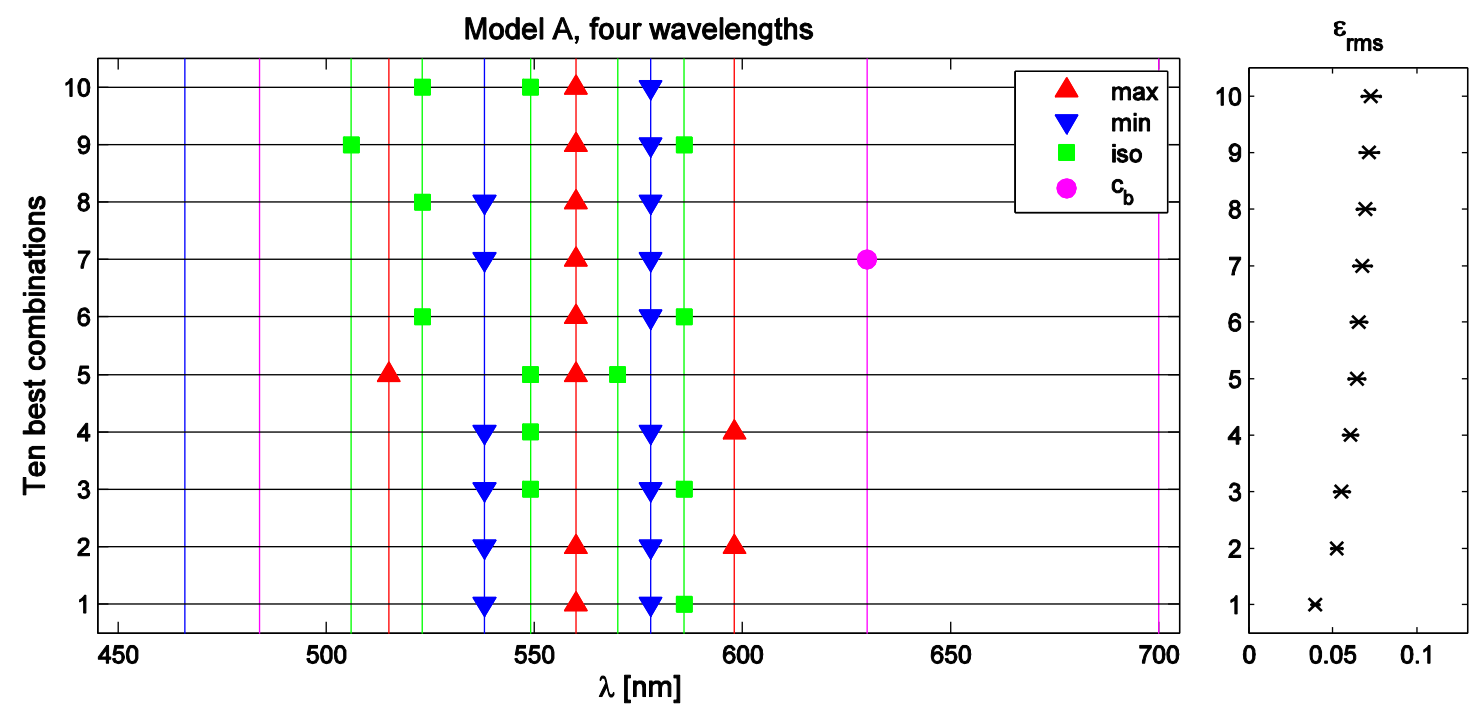

Fig. 6. The ten four-wavelength combinations with the lowest $\varepsilon_{\text {rms }}$ for model A. The vertical lines represent the 14 chosen wavelengths, the symbols on each horizontal line showing the four tested wavelengths. For the wavelength symbols, red upward-pointing triangle, blue downward-pointing triangle and the green square, see Fig. 1, for the magenta coloured dot, see Fig. 2 . The $\varepsilon_{\text {rms }}$ for each fourwavelength combination $\pm 2 \mathrm{SD}$ is shown in the right panel. 

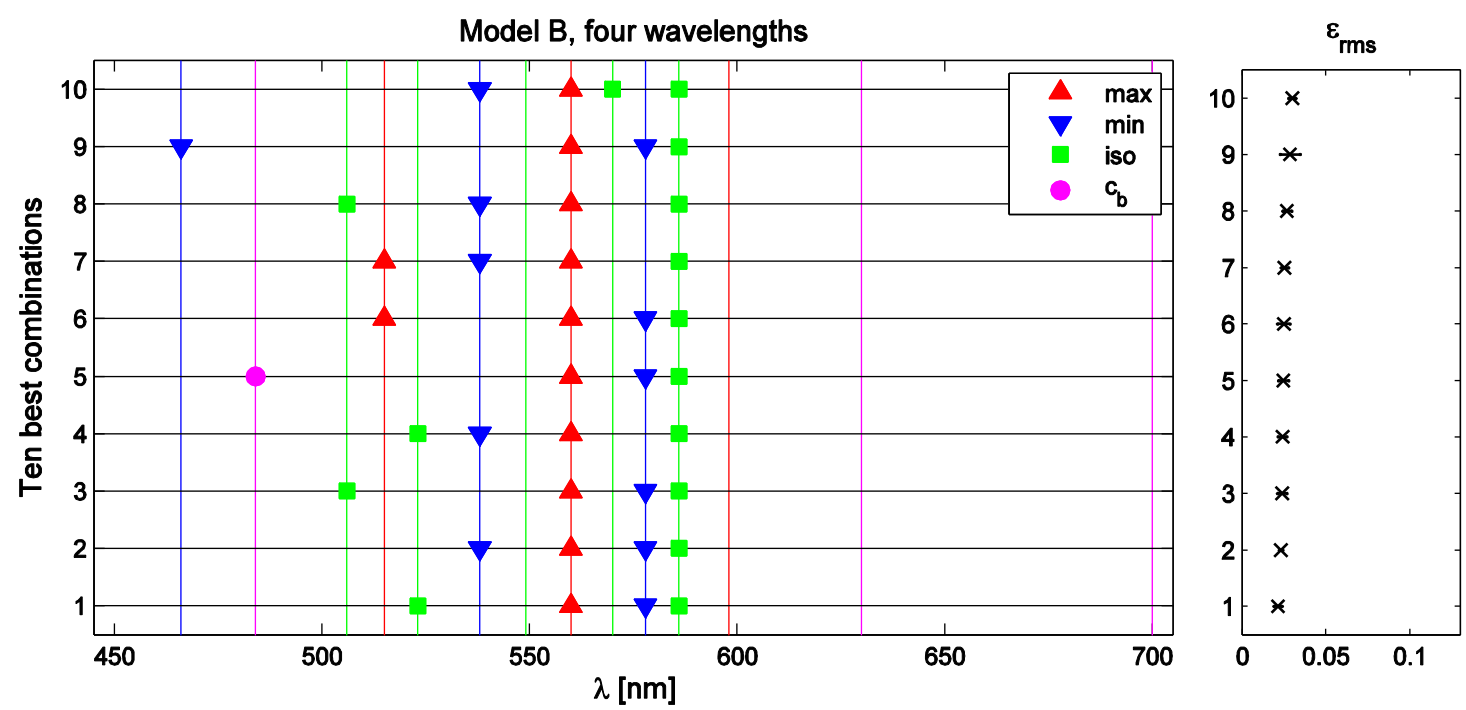

Fig. 7. The ten four-wavelength combinations with the lowest $\varepsilon_{\mathrm{rms}}$ for model B. The vertical lines represent the 14 chosen wavelengths, the symbols on each horizontal line showing the four tested wavelengths. For the wavelength symbols, red upward-pointing triangle, blue downward-pointing triangle and the green square, see Fig. 1, for the magenta coloured dot, see Fig. 2. The $\varepsilon_{\text {rms }}$ for each fourwavelength combination $\pm 2 \mathrm{SD}$ is shown in the right panel.

\section{DISCUSSION}

A range of Monte Carlo simulated spectra, originating from a two layer skin model, is evaluated in order to determine if the blood oxygen saturation can be determined from a reduced number of wavelengths. In total, 1000 simulated evaluation spectra, generated using randomly picked model parameters within a bio-optically relevant range, were assessed. The evaluation included both the number of selected wavelengths and the inverse model complexity for estimation of the blood saturation. The total number of 1000 assessed spectra was found sufficient to accurately determine the optimal selection of wavelengths.

Reviewing the wavelength selections giving the lowest estimation errors (Figs. 5-7), outline that the majority of the wavelengths are within the range 538-586 nm. Most of these selections contained the wavelengths $560 \mathrm{~nm}(\max ), 578 \mathrm{~nm}$ (min) and/or $586 \mathrm{~nm}$ (iso). For the 10 best ranked four-wavelength selections, all contained at least one min-point, one max-point and one isobestic point. Out of these selections, the combination $523 \mathrm{~nm}$ (iso), $560 \mathrm{~nm}, 578 \mathrm{~nm}$ and $586 \mathrm{~nm}$ produced the best result using the reduced model B with three free fitting parameters. The error for this combination was $2.2 \%$, which is close to the $1.6 \%$ error demonstrated by the complete model $\mathrm{C}$ using all 14 selected wavelengths (the combination that demonstrated the lowest estimation error).

The most complex inverse model (model C) is identical with the model used for randomly generating the 1000 spectra that are used for the evaluation. Even so, estimations based on model $\mathrm{C}$ cannot produce saturation estimations with an average error lower than $1.6 \%$ (using all 14 selected wavelengths). It is believed that this minimal error that deviates significantly from $0.0 \%$ is mainly caused by inverse solutions that are not globally optimal (i.e. the solution gets stuck in local minima close to the global minima). Model $\mathrm{C}$ produce the lowest error of all evaluated models only when evaluated using the 14 selected wavelengths.

It is evident that increasing the number of wavelengths when solving the inverse problem using any of the reduced models (A and B) does generally not result in improved estimates. In fact, four optimally selected wavelengths must be considered sufficient as this produced errors almost at the same level as model $\mathrm{C}$ using 14 wavelengths. Comparing the results from selecting four wavelengths shows that model $\mathrm{B}$ is the best selection with an error of $2.2 \%$ that is only marginally less accurate than model C (1.6\% error using 14 wavelengths). 
Increasing the number of selected wavelengths to 14 or above when applying model A or model B drastically increase the errors. For model A, these errors become even larger than if a fixed saturation of $50 \%$ in all evaluated spectra (error of $28 \%$ ) is assumed. If the number of selected wavelengths increases, it is evident that the model complexity (number of free model parameters) needs to increase as well, to be able to accurately estimate the blood saturation.

The result indicates that multi spectral imaging (MSI) systems only need four wavelengths for an accurate blood saturation estimation. Consequently, when using wavelength scanning MSI systems (e.g. filter wheel solutions and tunable filter solutions) the image acquisition speed can be improved significantly by only acquiring images for the optimal wavelengths. For other MSI systems where the different spectral planes are acquired in parallel (e.g. multi-camera solutions and on-chip pixel filtering solutions), decreasing the number of wavelengths will results in less complex hardware designs. From a data analysis perspective, it is also attractive to decrease the number of hyper planes and thereby lower the computational demand for the spectral analysis, potentially enabling real-time blood saturation imaging.

\section{CONCLUSIONS}

We have demonstrated that with only four optimally selected wavelengths, saturation estimation errors where on par with estimations based on 14 or more wavelengths, requesting computational needs and hardware solutions. Additionally, if the number of selected wavelengths are increased, the model complexity also needs to be increased to avoid poor estimations.

\section{ACKNOWLEDGEMENTS}

This project is supported by funding from the Centre for Industrial Information Technology (CENIIT) at Linköping University in collaboration with Perimed AB, Järfälla, and by the EU FP7 project SEMEOTICONS (SEMEiotic Oriented Technology for Individual's CardiOmetabolic risk self-assessmeNt and Self-monitoring), 611516.

\section{REFERENCES}

[1] R. R. Anderson, and J. A. Parrish, “The optics of human skin,” J Invest Dermatol, 77(1), 13-9 (1981).

[2] W. G. Zijlstra, A. Buursma, and W. P. Meeuwsen-van der Roest, "Absorption spectra of human fetal and adult oxyhemoglobin, de-oxyhemoglobin, carboxyhemoglobin, and methemoglobin,” Clin Chem, 37(9), 1633-8 (1991).

[3] I. Nishidate, T. Maeda, K. Niizeki et al., "Estimation of melanin and hemoglobin using spectral reflectance images reconstructed from a digital RGB image by the Wiener estimation method," Sensors (Basel), 13(6), 790215 (2013).

[4] M. Larsson, W. Steenbergen, and T. Stromberg, "Influence of optical properties and fiber separation on laser doppler flowmetry," J Biomed Opt, 7(2), 236-43 (2002).

[5] J. P. Ortonne, "Photoprotective properties of skin melanin," Br J Dermatol, 146 Suppl 61, 7-10 (2002).

[6] G. M. Palmer, and N. Ramanujam, "Monte Carlo-based inverse model for calculating tissue optical properties. Part I: Theory and validation on synthetic phantoms,” Appl Opt, 45(5), 1062-71 (2006).

[7] I. V. Meglinski, and S. J. Matcher, "Computer simulation of the skin reflectance spectra," Comput Methods Programs Biomed, 70(2), 179-86 (2003).

[8] T. Shi, and C. A. DiMarzio, "Multispectral method for skin imaging: development and validation," Appl Opt, 46(36), 8619-26 (2007).

[9] I. Fredriksson, [Quantitative Laser Doppler Flowmetry] Linköping University, Linköping Studies in Science and Technology. Dissertations, ISSN 0345-7524(2009).

[10] T. Igarashi, K. Nishino, and S. K. Nayar, "The appearance of human skin: a survey," Foundations and Trends in Computer Graphics and Vision, 3(1), 1-95 (2007).

[11] A. Krishnaswamy, and G. V. G. Baranoski, "A Biophysically-Based Spectral Model of Light Interaction with Human Skin ” Computer Graphics Forum, 23(3), 331-340 (2004).

[12] G. N. Stamatas, B. Z. Zmudzka, N. Kollias et al., "In vivo measurement of skin erythema and pigmentation: new means of implementation of diffuse reflectance spectroscopy with a commercial instrument," Br J Dermatol, 159(3), 683-90 (2008). 
[13] G. Lu, and B. Fei, "Medical hyperspectral imaging: a review," J Biomed Opt, 19(1), 10901 (2014).

[14] S. L. Jacques, "Optical properties of biological tissues: a review," Phys Med Biol, 58(11), R37-61 (2013).

[15] T. Lister, P. A. Wright, and P. H. Chappell, "Optical properties of human skin," J Biomed Opt, 17(9), 90901-1 (2012). 